\title{
Selected meeting abstracts of 2018 healthcare and cardiology conference
}

\author{
Bangkok, Thailand; September 18-19, 2018; Published: 16 Oct 2018
}

Correspondence to: Dr. Ahmed Ahmed Fouad Abdelwahab Ahmed, Consultant Cardiac Surgeon, Ain Shams University, 11566 Cairo, Egypt. E-mail: afouad38@yahoo.com

\section{Evaluation of the role of ischemia reversal therapy in ischemic heart disease using SPECT myocardial perfusion imaging: a pilot study}

\author{
Rohit Sane ${ }^{1}$, Rahul Mandole ${ }^{2}$
}

${ }^{1}$ Founder, Madhavbaug Cardiac Care Clinics and Hospital, Mumbai 400603, India. ${ }^{2}$ Department of R\&D, Madhavbaug Cardiac Care Clinics and Hospital, Mumbai 400603, India.

Ischemic heart disease (IHD) incidence has increased in India. Ayurveda, a 3000-year-old Indian traditional medicine system along with allopathic medicine can provide a solution to improve myocardial perfusion in stable IHD patients. This pilot study involves ischemia reversal programme (IRP), an Ayurvedic treatment modality to aid IHD patients using SPECT-myocardial perfusion imaging (SPECT-MPI) for assessment of myocardial perfusion in IHD patients. The present open-label study involved fourteen IHD patients who underwent IRP (21 sittings in total, administered twice per week) in Madhavbaug clinics along with their standard care therapy. The inclusion criteria were patients with known IHD, age $=40-70$ years, BMI $>20 \mathrm{~kg} / \mathrm{m}^{2}$, and stress test positive for inducible ischemia. SPECT-MPI was performed at enrolment and post-IRP (12-week follow-up) from December 2016 to September 2017. VO2max and time to onset of ischemia after stress test were also recorded. Seattle angina questionnaire (SAQ) was telephonically completed by research coordinators. Observations from SPECT-MPI test showed a significant difference in summed stress score $(13.5 \pm 10.3$, baseline vs. $10.7 \pm 10.1$, post-IRP; $P=0.01)$ as well as summed difference score $(8.9 \pm$ 6.2 , baseline vs. $6.2 \pm 6.3$, post- IRP; $P=0.03$ ) compared from baseline to post-IRP sittings. And we also observed the increase in $\mathrm{VO} 2 \mathrm{max}$ levels $(12.8 \pm 5.7$ to $19.4 \pm 7.8)$ and the time to onset of ischemia $(370.7 \pm 201.1$ to $597.8 \pm 201.9)$ was observed. SAQ scores showed significant improvement post-IRP $(30.2 \pm 3.6$ to $32.7 \pm 3.5)$. Findings of this study suggest an improvement in myocardial perfusion post-IRP in IHD patients and depict the positive role of IRP as an add-on to standard care therapy in IHD management.

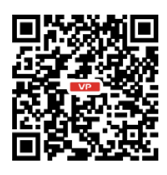




\section{Tachycardiomyopathy (a diagnosis not to be missed)}

\section{Gautam Singal}

Interventional Cardiologist, Holy Family Hospital, Okhla, New Delhi 110025, India.

Cardiomyopathy is a myocardial disorder in which the heart muscle is structurally and functionally abnor$\mathrm{mal}$ in the absence of coronary artery disease, hypertension, valvular disease, and congenital heart disease. Tachycardiomyopathy (TCMP) or arrhythmia induced cardiomyopathy is one such sub category and if adequately treated leads to improvement in LV function. It is defined as "Atrial and/or ventricular dysfunction - secondary to rapid and/or asynchronous/irregular myocardial contraction, partially or completely reversed after treatment of the causative arrhythmia". Arrhythmia may be the sole reason for LV dysfunction or is the reason for exacerbation in a patient with concomitant heart disease. In our case a 45-year-old lady (post MVR 1994) presented in emergency with sudden worsening dyspnea. At the time of admission she was in acute LVF with her ECG showing atrial fibrillation with fast ventricular rate. Left ventricular ejection fraction on echocardiogram was $35 \%$ with good prosthetic valve function. She underwent coronary angiogram, which showed normal coronaries with fluoroscopy showing good prosthetic valve function after stabilization and other precipitating causes were ruled out. She was cardioverted to normal sinus rhythm and was discharged from the hospital on guideline directed medical therapy. She is on regular follow-up and has remained asymptomatic and arrhythmia-free and her LV function has improved to 55\%. The incidence and prevalence of TCMP are uncertain. Atrial fibrillation (AF) is present in $10 \%$ to $50 \%$ of patients with HF; many patients with cardiomyopathy and AF have worsening symptoms and LV function solely due to poorly controlled ventricular rates. The mechanisms of TCMP are not fully defined but include subclinical ischemia, abnormalities in energy metabolism, redox stress and calcium overload. The possibility of TCMP should be considered when eliciting a history of any new diagnosis of LV dysfunction, if there is evidence of persistent or frequently occurring tachycardia and its timely diagnosis is important given the potential for near-complete recovery with appropriate treatment.

\section{Comparison of outcomes of thrombolysis vs. re-preoperation for stuck prosthetic valve in mitral position - 10 year experience}

\section{Karthik Raman, Anbarasu Mohanraj, Ravi Agarwal, Ejaz Ahmed Sheriff, Kurian Valikapathalil Mathew Kurian, Rajan Sethuratnam}

Department of CTVS, Madras Medical Mission, Chennai, Tamil Nadu 600037, India.

Aim: To analyse the outcomes of thrombolysis vs. re-preoperations for stuck prosthetic valves in mitral position.

Methods: From the time period of January 2005 till December 2015, a total of 36 patients had undergone thrombolysis and 31 patients had undergone re-preoperations for stuck prosthetic valves in mitral position in our institution. The follow up period was 225 patient-years in thrombolysis group and 208 patient-years in re-operative group. The prosthetic sizes were $25,27,29,31$ in mitral position. Mean functional class was $2.6 \pm$ 0.8 in thrombolysis and $3.4 \pm 0.9$ in re-operation group. The peak and mean gradient was $37.5 \pm 4.8,18.2 \pm 3.56$ in thrombolysis and $40 \pm 2.7,20 \pm 4.3$ in re-operation group respectively. Transthoracic and transoesophageal echocardiographs along with fluoroscopy were done for all the patients. The causes of obstruction were pannus formation in 7, generation of thrombus in 50, and both pannus and thrombus in 10. Presence of pannus was an indication for surgery. 
Results: The analysis of thrombolytic group is as follows: incidence of death was $2 \%$ per patient-year, freedom from Peripheral embolism $97.3 \% \pm 1.8 \%$, freedom from CNS bleeding $98.2 \% \pm 3.8 \%$, freedom from stroke $97.2 \% \pm 2.6 \%$, freedom from TIA $98.1 \% \pm 2.8 \%$, freedom from Coronary embolism $100 \%$, freedom from major bleeding with transfusion $96.3 \% \pm 4.8 \%$, freedom from thrombolytic failure $95.4 \% \pm 3.7 \%$. The peak and mean gradient was $11.5 \pm 4.8,5.2 \pm 3.56$ at the end of completed thrombolysis. The analysis of re-operative group are as follows: incidence of death was $3 \%$ per patient-year, freedom from Peripheral embolism $98.3 \% \pm 4.8 \%$, freedom from CNS bleeding $99.2 \% \pm 2.8 \%$, freedom from stroke $95.2 \% \pm 3.5 \%$, freedom from TIA $100 \%$, freedom from Coronary embolism 100\%, freedom from major bleeding with transfusion $94.3 \% \pm$ $5.4 \%$. The peak and mean gradient was $9.5 \pm 4.8,4.2 \pm 3.56$ at the end of re-operation on the 7 th post-op day, $10.5 \pm 4.8,4.2 \pm 1.56$ at the end of first year and $11.5 \pm 4.8,5.2 \pm 3.75$, at the end of third year respectively.

Conclusion: Re-preoperation and thrombolysis are the widely accepted options for treatment of mechanical heart valve thrombosis and both seem to be equally effective. The percentage of embolic events and recurrent thrombosis are higher in thrombolysis group while we had almost comparable mortality in both the groups. Longer follow-up with a large group of patients is necessary for further results.

\section{Outcome of coronary artery bypass grafting surgery in patients with low ejection fraction}

\section{Mohammed Aslam Hossain, Mayank Acharya, Dharmendra Joshi, Niraj Bhattarai, Satish Vaidya, Karan Rai, Sanaul Sarker, Samir Azam Sunny}

Department of Cardiac Surgery, Bangabandhu Sheikh Mujib Medical University, Shahbag, Dhaka 1000, Ban-
gladesh.

Aim: To analyze the outcome of coronary artery bypass grafting surgery in patients with low ejection fraction. Coronary artery bypass surgery is one of the most frequently performed among various surgeries. In recent years the mortality and morbidity related to the procedure has decreased even in the developing world. However a significant peri surgical morbimortality burden remains among patients with heart failure patients. The factors of this may be due to problems in wound healing, pulmonary complications, post perfusion syndrome, poor tissue oxygenation, acute liver and kidney injuries, prolonged ventilation, frequent cerebrovascular events, presence of other comorbid conditions, all of which affect the patient more than compared to a similar patient with moderate or normal preoperative ejection fraction.

Methods: Data were obtained and analyzed from 102 patients who underwent coronary artery bypass grafting surgery having a preoperative left ventricular ejection fraction $\leq 40 \%$. A subgroup of patients with $\mathrm{EF} \leq$ $30 \%(n=28)$ were also analyzed separately.

Results: The mean age of our patient was $57.9 \pm 7.5$ years of which $85(83.3 \%)$ were males and 17 (16.7\%) were females. 74 (72.5\%) patients had LVEF $31-40$ and $28(27.5 \%)$ had $\leq 30$. Comparison of pre-operative LVEF and post-operative LVEF at 1 and 3 months revealed mean LVEF 33.15 against $37.63(P \leq 0.001)$ at 1 month and 33.09 against $38.04(P \leq 0.001)$ at 3 months. Other per- and post-operative outcome variables were also analyzed like arrhythmia, inotrope support time, bypass requirement, pulmonary complications, mechanical ventilation time, death and other variables.

Conclusion: Operations on patients with low ejection fraction warrant additional vigilance, albeit with proper precaution the outcome is favorable. 


\title{
5. Rare case of multiple right atrial myxomata with acute pulmonary thromboembolism: a case report
}

\author{
Parin Chandrakant Sangoi, Ramkumar Rajaram, Vignesh Gomathinayagam, Ajit Mullasari \\ Department of Cardiology, Madras Medical Mission, Chennai 600037, India.
}

This is a case report of a 56-year-old morbidly obese female who was presented to our emergency room with complaints of acute onset progressive breathlessness since 3 days (NYHA class IV) with presyncope and palpitation but no history of chest pain. The patient was diagnosed with pulmonary thromboembolism with IVC - RA thrombus 4 years back. During examination she was found to be morbidly obese, with presence of skin tags and naevi in interscapular and left zygoma region. Her vitals were: HR 118/min, BP 100/70 mmHg and $\mathrm{SPO}_{2} 85 \%$ in room air. Cardiopulmonary examination revealed good heart sounds with basal crepts. ECG showed Sinus tachycardia (+). Echo revealed a pedunculated right atrial (RA) mass attached to the lower intra atrial septum of size $2.9 \mathrm{~cm} \times 1.5 \mathrm{~cm}$ protruding through the tricuspid valve in diastole. Another mass of size $1 \mathrm{~cm} \times 2 \mathrm{~cm}$ was seen in Right Atrium with pedicle attached near IVC-RA junction. The patient also had severe Tricuspid Regurgitation with pulmonary hypertension. Further investigations revealed an elevated D - Dimer and CT PA confirmed RA mass with acute pulmonary embolism involving both the lungs. The patient was treated with low molecular weight heparin and follow-up echo showed decrease in Pulmonary artery pressure with improvement in her symptoms. The presence of morbid obesity, cushingoid habitus, cutaneous skin tag in the left interscapular area, cutaneous neavi, multiple atrial myomata and pulmonary embolism together steered us towards the possible diagnosis of Carney's Complex. This case has been presented because the right atrial mass produces a diagnostic dilemma with differential diagnosis being thrombus (type $\mathrm{A} \& \mathrm{~B}$ ), primary tumor of heart commonly myxomas, lipomas, sarcomas and metastatic tumours. Hence it is important to differentiate the masses because treatment options and prognosis of each lesion vary.

\section{Lectin-like oxidized-LDL receptor as the anti-atherosclerosis vaccine candidate}

\section{Valentina Yurina}

Brawijaya University, Malang 65145, Indonesia.

Cardiovascular disease remains the most burdening health problems worldwide. The disease accounts for $31.43 \%$ mortality globally. Some of the major risk factors for the disease are hypertension (33\%), hypercholesterolemia (19\%), overweight (56\%), and current smoking (19\%). Hypercholesterolemia indicated by the elevated low-density lipoprotein (LDL) cholesterol and its oxidized products, which unlike the LDL cholesterol, is unrecognizable by its receptor. The oxidized LDL cholesterol (ox-LDL) is taken up by the scavenger receptors in the endothelial cells and macrophages. Lectin-like ox-LDL receptor-1 (LOX-1) remains as the major oxLDL scavenger receptor in the endothelial cells. The LOX-1 participates in the early atherosclerosis phase by inducing the endothelial dysfunction. However, in the late stage of atherosclerosis, LOX-1 also plays a role in the smooth muscle cells apoptosis and foam cells formation. The LOX-1 expression is relatively low in the basal level, hence its expression is enhanced by several clinical conditions, such as hypertension, diabetes, obesity, atherosclerosis, and myocardial infarction. Several approaches demonstrated that the inhibition of LOX-1 reduces the endothelial dysfunction and atherosclerosis development. These studies indicated that LOX-1 inhibition is a promising candidate for atherosclerosis prevention. Our study demonstrated LOX-1 efficacy as the atherosclerosis vaccine candidate through DNA vaccination and subunit vaccination approach. 